\title{
Differential Diagnosis of HyperCKemia
}

\section{(ㄷ) (i) (우 $\ominus$}

Authors

Rudolf A. Kley ${ }^{1}$, Tobias Schmidt-Wilcke ${ }^{2,3}$, Matthias Vorgerd ${ }^{1}$

\section{Affiliations \\ 1 Heimer Institute for Muscle Research, Department of Neurology, BG University Hospital Bergmannsheil, Ruhr University, Bochum, Germany \\ 2 Department of Neurology, St. Mauritius Therapieklinik Meerbusch, Germany \\ 3 Institute of Clinical Neurosciences and Medical Psychology, Heinrich-Heine University of Düsseldorf, Germany}

Keywords

creatine kinase, CK elevation, myopathy, neuropathy, algorithm

Bibliography

DOI https://doi.org/10.1055/s-0043-124361

Neurology International Open 2018; 2: E72-E83

(c) Georg Thieme Verlag KG Stuttgart · New York

ISSN 2511-1795

Correspondence

Prof. Dr. med. Rudolf Andre Kley

Heimer Institut für Muskelforschung

Neurologische Universitäts- und Poliklinik

Berufsgenossenschaftliches Universitätsklinikum

Bergmannsheil

Bürkle-de-la-Camp-Platz 1

44789 Bochum

Germany

rudolf.kley@rub.de

\section{ABSTRACT}

Elevated serum creatine kinase (CK) activity is usually an indicator of muscle damage. HyperCKemia is often an incidental finding and should be controlled after refraining from physical activity for some days, especially in asymptomatic patients. Furthermore, data from recent studies indicate that the upper limits of normal (ULN) need to be revised upward. This review includes an algorithm for the differential diagnosis of CK elevation in patients without muscular symptoms. In the field of neurology, in particular myopathies and neuropathies with affection of the lower motor neuron can cause symptomatic hyperCKemia, with CK values $>1000 \mathrm{U} / \mathrm{I}(16,7 \mu \mathrm{kat} / \mathrm{l})$ being indicative of a primary muscle disorder. Diseases with very high CK values include subtypes of muscular dystrophies, idiopathic inflammatory myopathies and metabolic myopathies. However, a normal or only slightly elevated CK value does not exclude the presence of a myopathy. The individual diagnostic procedure (e. g., muscle imaging, special laboratory studies, muscle biopsy and genetic testing) depends on the clinical phenotype and the results of electrophysiological studies. HyperCKemia can also be an adverse effect of several drugs including statins. In asymptomatic patients, statin-associated CK elevations $<5$ times the ULN can be tolerated. In patients with higher CK values and/or muscle symptoms, LDL-cholesterol lowering therapy should be changed. Rhabdomyolysis is a potentially life-threatening condition and is accompanied by highly elevated CK values. Acute phase treatment includes preserving renal function and restoring metabolic derangements.

\section{Introduction}

Creatine kinase (CK) is an enzyme playing an important role in the energy metabolism of cells. It catalyzes the transfer of a phosphate from phosphocreatine to ADP, thus supporting the resynthesis von ATP. CK activity is by far the highest in skeletal muscle [61]. Total CK activity, measured $\mathrm{N}$-acetylcysteine (NAC)-stabilized in serum or plasma (CK-NAC), comprises the activities of the various CK isoenzymes (CK-MM: skeletal muscle type, percentage of total activity normally >93\%; CK-MB: myocardial band, percentage of total activity normally < $6 \%$; CK-BB: brain type, percentage of total activity up to a maximum of about $1 \%$; CK-MiMi: mitochondrial type; usually not detectable in serum). Increased CK levels typically indicate muscle damage with disruption of sarcolemmal integrity and may be caused by a variety of conditions ( $\downarrow$ Table 1 ). Here, an exemption is macro-CK. In macro-CK type 1 (not associated with disease), immunoglobulins bind to CK-BB, while in macro-CK type 2 (usually associated with significant disease) oligomeric mitochondrial CK is present. In macro-CK, the CK-MB percentage of total CK is usually $>25 \%$, due to the method used. This helps to distinguish it from myocardial infarction where the CK-MB percentage is typically between $6 \%$ and $20 \%$ (for total CK levels of up to approximately $7500 \mathrm{U} / \mathrm{L}[125 \mu \mathrm{kat} / \mathrm{L}])$. 
- Table 1 Causes of hyperCKemia (examples).

Inherited and acquired myopathies

Inherited and acquired neuropathies (including motor neuron diseases, radiculopathies)

Medications (e. g., statins, fibrates, beta blockers, angiotensin-II receptor blockers, clozapine, hydroxychloroquine, isotretinoin, colchicine)

Physical exertion, sports

Trauma (e.g., fall, crush injury)

Seizures

Muscle cramps (paraphysiological or symptomatic)

latrogenic (e. g., intramuscular injection, EMG examination, surgery)

Pregnancy

Ethnicity

Surgical procedures

Toxins (e. g., alcohol, heroin, cocaine)

Endocrine (e. g., diabetes mellitus, hypothyroidism, hypoparathyroidism, acromegaly)

Viral infections

Metabolic (hypokalemia, hyponatremia, hypophosphatemia)

Myocardial infarction / chronic cardiac disease

Obstructive sleep apnea syndrome

Neuroacanthocytosis

macro-CK

Renal disease

Celiac disease

Connective tissue disorders

Malignancies

CK level is an important parameter in the differential diagnosis of neuromuscular diseases. Yet, hyperCKemia as an incidental finding is of limited diagnostic significance and should always be evaluated in the context of clinical findings. Special attention should be paid to the fact that after physical activity, e. g., when exercising or on the job, and after injuries, it is normal to find significant increases in serum CK activity over a period of several days. In particular after extreme physical exertion, it may take more than 2 weeks until CK levels have returned to normal [42]. Thus, it is advisable to repeat CK testing after several days of rest. Key factors influencing the further diagnostic process include the patient's medical history and clinical examination findings.

\section{What is Still Normal? - The Reference Range Problem}

When do we speak of hyperCKemia? This is not a question that can easily be answered as the answer depends on the reference values used - and these still are the subject of ongoing discussions. The International Federation of Clinical Chemistry and Laboratory Medicine (IFCC) last updated the standards for measuring serum CK activity in 2002 [54]. The "preliminary" upper limits of normal (ULN) issued then were $145 \mathrm{U} / \mathrm{L}(2.4 \mu \mathrm{kat} / \mathrm{L})$ and $171 \mathrm{U} / \mathrm{L}(2.9 \mu \mathrm{kat} / \mathrm{L})$ for women and men, respectively. These values should represent the $97.5^{\text {th }}$ percentile which would mean that only $2.5 \%$ of healthy individuals should have CK levels above this upper limit. These normal values are based on IFCC recommendations from 1991 [20] which in turn are derived from national recommendations of various European countries between 1976 and 1982 [20]. However, attention should be paid to differences in the method used to measure CK levels and the studied patient population. In addition, normal ranges were calculated based on the assumption of normal distribution. Yet, there is some evidence indicating that this not the case, especially if CK levels are high [38]. Ultimately, this leads to an increased number of false positive results. For example, in a study with 968 healthy subjects younger than 25 years of age conducted in Israel, $18.9 \%$ of men and $4.6 \%$ of women had CK levels $>200 \mathrm{U} / \mathrm{L}$ (3.3 $\mu \mathrm{kat} / \mathrm{L})$ [28]. In a study by Brewster et al. from the Netherlands published in 2007, which enrolled 1444 persons aged between 34 and 60 years, $36 \%$ had elevated CK levels (with ULNs of $140 \mathrm{U} / \mathrm{L}(2.3 \mu \mathrm{kat} / \mathrm{L})$ for women and $174 \mathrm{U} / \mathrm{L}(2.9 \mu \mathrm{kat} / \mathrm{L})$ for men $)$ [6]. Here, the ethnicity of the subjects was the decisive factor. Among subjects of European origin, only $8 \%$ of women and $17 \%$ of men had elevated CK levels. By contrast, among subjects of African origin, $42 \%$ of women and even as much as $62 \%$ of men were found to have increased CK levels. The $97.5^{\text {th }}$ percentiles for serum CK activity were $201 \mathrm{U} / \mathrm{L}(3,4 \mu \mathrm{kat} / \mathrm{L})$ in European women and $322 \mathrm{U} / \mathrm{L}$ $(5.4 \mu \mathrm{kat} / \mathrm{L})$ in European men and thus significantly higher than the IFCC's ULNs. In subjects of African origin, 97.5 $5^{\text {th }}$ percentiles were once again twice as high with $414 \mathrm{U} / \mathrm{L}(6.9 \mu \mathrm{kat} / \mathrm{L})$ for women and $801 \mathrm{U} / \mathrm{L}(13.4 \mu \mathrm{kat} / \mathrm{L})$ for men.

These numbers highlight the need to further discuss the reference range for CK. The 2010 guidelines of the European Federation of Neurological Societies (EFNS) propose to adapt the CK reference range according to the study by Brewster et al. [25]. However, in 2012 a Norwegian study was published which included data of altogether 6904 persons. The $97.5^{\text {th }}$ percentiles of CK was $207 \mathrm{U} / \mathrm{L}$ (3.5 $\mu \mathrm{kat} / \mathrm{L})$ for women and $367 \mathrm{U} / \mathrm{L}(6.1 \mu \mathrm{kat} / \mathrm{L})$ for men [31]. As this was a high-quality study with significantly more subjects, we advocate relying on these data as a guidance for individuals of European origin. Amending the reference range by applying higher cut-off values results in improved specificity for neuromuscular disorders and significantly reduces the number of false-positive results. It helps to prevent unnecessary additional diagnostic tests in health individuals. On the other hand, it lowers the sensitivity of the laboratory parameter. However, this downside appears to be of comparatively low clinical relevance [41]. It should be borne in mind that even with the current reference range not all neuromuscular disorders are associated with elevated CK levels. Thus, in the presence of clinical signs and symptoms indicative of neuromuscular abnormalities, an adequate work-up should be performed in all cases, regardless of CK levels.

\section{Asymptomatic HyperCKemia}

In asymptomatic hyperCKemia, the patient has no history of relevant muscular symptoms and, by definition, clinical neurological examination is unremarkable. The causes of this finding are many and varied ( $>$ Table 1). Here, key questions to be addressed in the medical history include previous illnesses, neuromuscular disor- 
ders and adverse events from anesthesia in the patient's family as well as the alcohol and medication history. Before initiating further investigations, CK levels should be re-checked. A large study with more than 12000 participants found that when initially elevated CK levels were re-assessed during a standardized follow-up examination (avoidance of excessive physical activity and no alcohol consumption within 3 days prior to blood collection), CK levels had returned to normal in almost $70 \%$ of cases [30]. In more than $40 \%$ of the remaining cases, the cause identified was not within the spectrum of neuromuscular disorders. The most common cause of elevated CK levels was statin treatment, followed by thyroid disease, renal disease and cardiac disease [30]. Thus, in patients with asymptomatic elevation of CK levels, the diagnostic work-up should initially focus on potential medical causes. In patients with persistent or recurrent hyperCKemia not explained otherwise ( $\triangleright$ Table 1 ), the differential diagnosis should in particular include the following conditions:

- idiopathic hyperCKemia not associated with disease

- inherited or acquired neuromuscular disease (NMD) prior to

clinical manifestation

- isolated hyperCKemia with mutation(s) in an NMD gene (also female carriers/heterozygote carriers of a recessive disease)

Increased serum CK activity appears to be not harmful as such and consequently it is not associated with disease. In case of severe damage to muscle tissue, it is not the released CK, but mainly the released myoglobin which causes renal injury (see below). However, finding the cause of hyperCKemia is desirable as it is a prerequisite for adequate counselling of the patient. When followed up over a certain period of time, prognosis is generally good $[9,46]$. However, there is no guarantee that the patient will not develop clinical manifestation of a neuromuscular disorder later in the course of the disease; therefore, follow-up examinations at wider intervals (every 1 -2 years) are advisable. Another important aspect is that even in asymptomatic cases of hyperCKemia, patients have an increased risk for a genetic predisposition to develop malignant hyperthermia (MH). This dreaded complication of anesthesia is associated with increases in body temperature and CK levels, typically accompanied by mutations in the RYR1 gene $[34,63]$. Consequently, when patients with unexplained elevation of CK levels undergo anesthesia, substances which may trigger $\mathrm{MH}$ (inhalational anesthetics and depolarizing muscle relaxants) should be avoided as a precaution.

\section{How successful is the diagnostic work-up for asymptomatic hyperCKemia?}

In recent years, several retrospective studies addressing this issue have been published $[8,15,45,16,55,34,46]$. Here, it has to be taken into account that not only data of asymptomatic patients, but also of patients with mild unspecific symptoms were included. The results of these studies varied for a number of reasons [25]. However, looking at the overall picture it can be stated that about half of the patients had abnormal EMG findings. With extensive further work-up, including muscle biopsy and genetic tests, $20 \%$ of patients were diagnosed with myopathy and 5-10\% with neuropathy. In approx. $40 \%$ of patients, histology revealed unspecific muscle tissue changes which could not be more precisely linked to spe- cific conditions. However, it is likely that if the now available advanced molecular genetic tests (panel diagnosis, whole exome sequencing) were used, mutations with known disease genes could be demonstrated in a subgroup of these patients. About one third of patients had no abnormalities at all and was classified as idiopathic hyperCKemia.

\section{Recommendations for the diagnostic algorithm in patients with asymptomatic hyperCKemia}

- Fig. 1 shows an algorithm established from a pragmatic point of view based on the available evidence and existing guidelines. As described above, the first step should be to measure CK levels after several days with little physical activity and to determine the percentage of CK-MB (especially with regard to potential macro-CK). In case of persistent increased CK activity it is advisable to first rule out medical causes or side effects of drugs. If this does not clearly indicate a diagnosis, the EFNS guidelines recommend to only initiate further neurological work-up if CK levels exceed 1.5 times the upper limit of normal. However, we think that the use of $\mathrm{MH}$-triggering substances should be avoided as a precaution, even in cases with mild hyperCKemia. With regard to further diagnostic tests, we consider it advisable to perform an MRI scan of the leg muscles (with an interval to EMG), besides electrophysiological testing, as this method is very sensitive in detecting lipomatous and edematous muscle changes. In case of unremarkable results, a suspected diagnosis of idiopathic hyperCKemia can be established and subsequent diagnostic assessments be limited to follow-up checks (with the residual risk of wrong classification; see Info Box Genetic testing in asymptomatic hyperCKemia). However, if findings are indicative of myopathy or neuropathy, further testing has to be performed as required. In case of specific findings, e. g., detection of myotonic discharge series in EMG, or in case of a specific distribution pattern of muscle alterations in MR imaging, a targeted genetic assessment may be indicated. If findings are suggestive of myopathy, it is recommended to perform a muscle biopsy; in this case, histopathological processing and analysis should be performed by an experienced center. If specific histological changes are detected, it may be possible to establish a diagnosis or initiate targeted genetic examinations. In case of unspecific myopathological changes, stepwise diagnosis as in symptomatic hyperCKemia should be considered. If histology is unremarkable, the further diagnostic procedure is to be established on an individual basis, taking into account the extent of the changes in electromyography and MRI.

\section{Symptomatic HyperCKemia}

Patients with symptomatic hyperCKemia report muscle problems and usually show abnormalities on clinical neurological examination. With regard to potential causes, our focus is on acquired and inherited myopathies and neuropathies. It is beyond the scope of this review to discuss the approximately 800 different neuromuscular disorders with now more than 250 known disease genes in detail. In addition, the available evidence on these rare diseases is often inadequate and CK levels may vary widely, even between patients with the same condition. Thus, the information provided in the following example is only indicative. 
no further work-up

$\downarrow$

recheck CK levels + CK-MB

$\leftarrow$ no - after reduced physical activity

$\rightarrow$ CK levels still elevated?

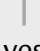

yes

$\checkmark$

explained by medical

condition or drug treatment?

treatment of the underlying

and

no

$\downarrow$

suspicion of idiopathic

$\leftarrow$ no - CK increased $>1.5$

hyperCKemia

$\rightarrow$ monitor clinical course; further work-up in case of significant increase in CK levels or CK elevation $>10$ times

$\rightarrow$ in case of anesthesia, avoid the use of substances that can trigger malignant hyperthermia (inhalational anaesthetics and $\leftarrow$ no depolarizing muscle relaxants)

EMG/muscle MRI; if required, ENG $\rightarrow$ abnormalities detected?

evidence of myopathy

$\downarrow$

specific changes? yes $\rightarrow$ targeted genetic tests<smiles>[TeH]</smiles>

no

$\downarrow$

muscle biopsy $\rightarrow$ result?

specific changes?

evidence

neuro-
pathy

further work-up (see also Dräger

disease, switch of medication,

if required 4/2016)

diagnosis established or targeted genetic testing unspecific changes stepwise diagnosis as in symptomatic hyperCKemia, if needed

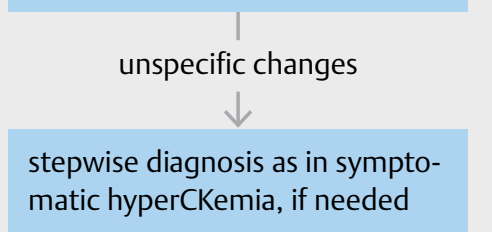

- Fig. 1 Diagnostic algorithm for asymptomatic hyperCKemia. NMD: neuromuscular disease; CK: creatine kinase; EMG: electromyography; ENG: electroneurography.

\section{Myopathies}

Progressive muscle weakness, in some disorders with characteristic distribution, in conjunction with muscle atrophy is the hallmark of most primary muscle disorders. Facultative signs and symptoms, such as myalgia (see Info Box), exercise intolerance, myotonia or signs of increased muscle excitability, rash, joint contractures, dysmorphic stigmata, and signs of multi-organ involvement, may provide valuable information for establishing the diagnosis. > Fig. 2 shows examples of some patients with various myopathies. For recommendations on the diagnostic process in myopathies, please refer to the recently updated and open-access guideline of the German Society of Neurology (DGN) on the topic (AWMF registration number 030/115). In some myopathies, phenotypes are so characteristic that the diagnosis established after laboratory testing and electrophysiological studies can usually be confirmed by direct genetic testing. These disorders include facioscapulohumeral muscular dystrophy and oculopharyngeal muscular dystrophy, myotonic dystrophy type 1 (and to some extent also type 2), and certain types of non-dystrophic myotonias. By contrast, the differential diagnostic work-up of other myopathies often requires 

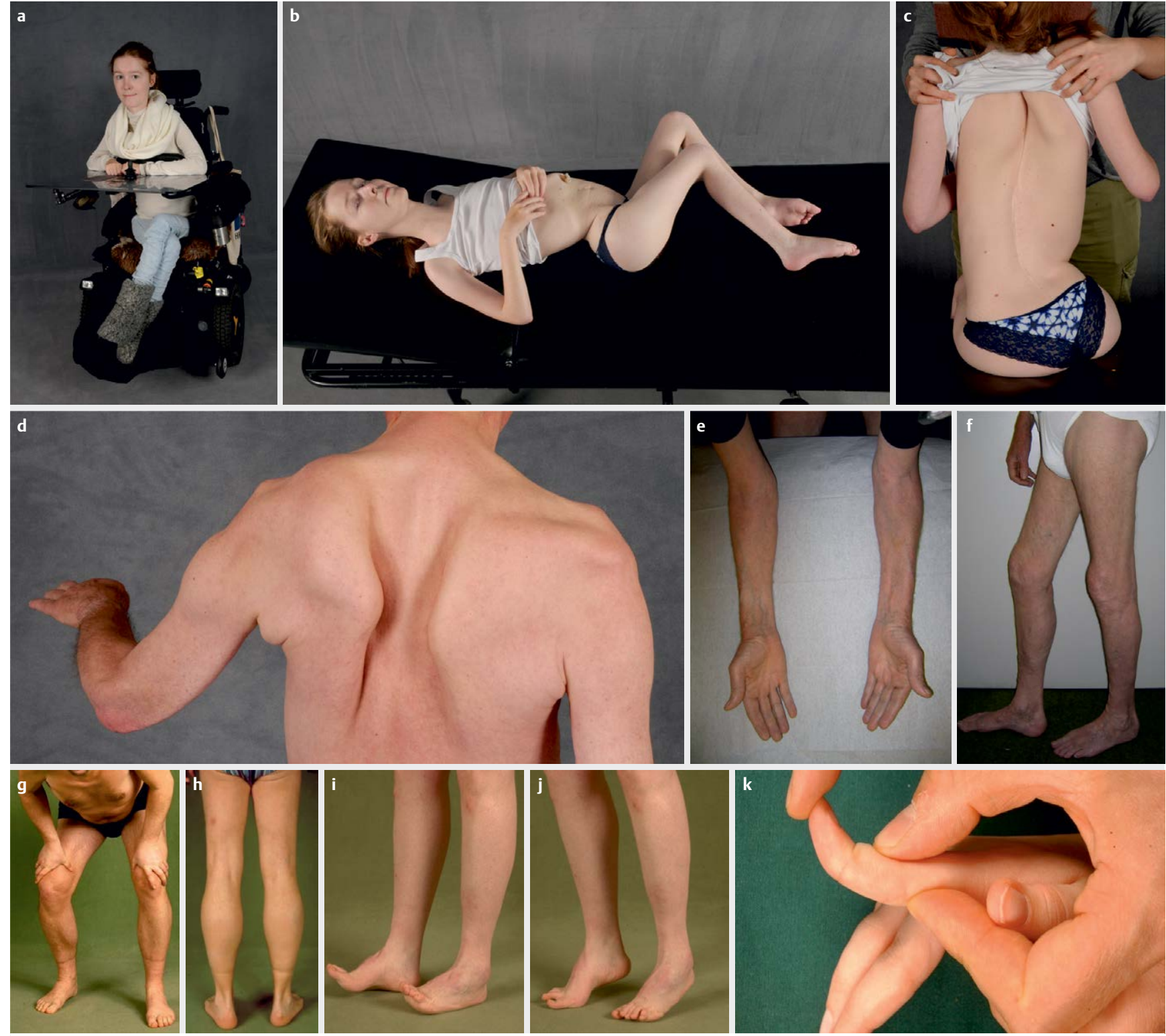

Fig. 2 Examples of patients with myopathies. a-c: 23-year-old patient with muscular dystrophy of unclear etiology, presenting with severe, more proximal paralysis with muscular atrophy and bilateral scapular winging, contractures of joints, scoliosis, and status post PEG placement. The patient requires non-invasive mechanical ventilation because of respiratory muscle involvement. d: Typical picture of facioscapulohumeral muscular dystrophy with marked scapular winging and poly-hill sign. e-f: Paresis and atrophy of flexor digitorum profundus muscles and the quadriceps femoris muscle in patient with sporadic inclusion body myositis. g: Gowers' sign (walking hands up the thighs in order to stand up) in a patient with limb-girdle muscular dystrophy type $2 \mathrm{~A}$. h: Becker muscular dystrophy with pseudohypertrophy of calf muscles. i-j: Distal myopathy caused by CAV3 mutation with marked paresis of foot dorsiflexors. k: Bethlem myopathy with joint hypermobility.

extensive and sophisticated special diagnostic tests, among others muscle MRI ( $\triangleright$ Fig. 3) and muscle biopsy ( $>$ Fig. 4). Therefore, it is advisable to refer these patients to a specialized neuromuscular center. Patients should be informed that it is not always possible to identify the cause of the disease even after extensive diagnostic testing. Using the diagnostic resources available today, a precise diagnosis can be established in up to $75 \%$ of symptomatic cases [43]. However, if the most common causes have already been ruled out, chances of successfully establishing the diagnosis may be considerably poorer in some patients. On the other hand, advanced genetic tests have opened up new possibilities which have led to the identification of numerous new disease genes in recent years. Thus, re-evaluation of still undiagnosed patients with symptomatic hyperCKemia at a later point may well be justified. Special attention should be paid to disorders for which specific treatment option are available or will become available in the foreseeable future. A noteworthy example is Pompe disease which can be treated with enzyme replacement therapy.

Inherited and acquired myopathies are often, but not always, associated with elevated CK levels. Usually, it is not possible to draw direct conclusions on the underlying cause of a myopathy based on CK levels alone. However, taking into account the patient's (fam- 

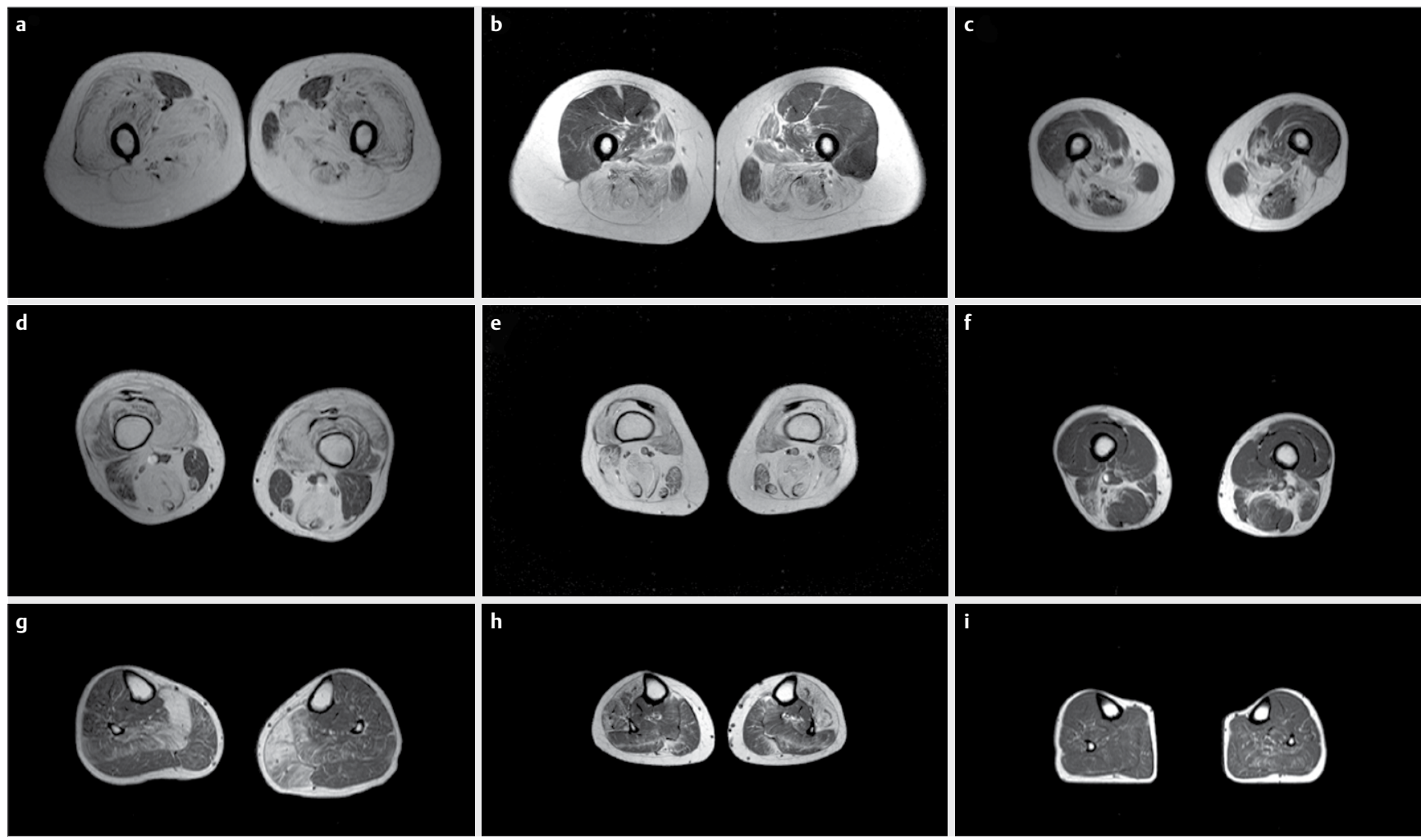

- Fig. 3 T1-weighted MRI scan of leg muscles in patients with limb-girdle muscular dystrophies. a/d/g: LGMD2A (calpain-3 defect); b/e/h: LGMD2R (recessive desmin defect); c/f/i: LGMD2I (FKRP defect). Differences in the pattern of lipomatous muscle alterations are noted. In the calf region, patients with LGMD2A, for example, show asymmetric marked fatty degeneration primarily of the soleus and the medial gastrocnemius muscles, while in LGMD2R patients the tibialis anterior, extensor digitorum longus and peroneal muscles are primarily affected. In the thigh region, for example, with LGMD2I the semitendinosus and semimembranosus muscles are less involved compared to the other 2 subtypes of LGMD.

ily) history, clinical phenotype and results of additional diagnostic tests, the extent of hyperCKemia can influence the direction of the further diagnostic work-up. Disorders associated with very high CK levels include certain types of muscular dystrophies, idiopathic inflammatory myopathies and metabolic myopathies.

Muscular dystrophies are a good example to demonstrate the wide range of elevated CK levels in myopathies. In patients with mutations in the dystrophin gene, the most common cause of hereditary myopathy (Duchenne and Becker types of muscular dystrophy), mean CK levels are about $10000 \mathrm{U} / \mathrm{L}$ (166.7 $\mu \mathrm{kat} / \mathrm{L})$ [64]. In patients with limb-girdle muscular dystrophy (LGMD), certain recessive subtypes, e. g., LGMD2B (affected protein: dysferlin), LGMD2I (FKRP) and LGMD2L (anoctamin 5), are associated with high CK levels in the 5-digit range. By contrast, in other subtypes of the disease, e. g., LGMD1A (myotilin), LGMD1D (DNAJB6) or LGMD2] (titin), CK levels are only slightly ( $<600 \mathrm{U} / \mathrm{L} ;<10 \mu \mathrm{kat} / \mathrm{L}$ ) to moderately (600-1500 U/L; 10-25 $\mu \mathrm{kat} / \mathrm{L}$ ) increased or even within the normal range. This also applies to facioscapulohumeral muscular dystrophy (FSHD) and myotonic dystrophies, the most common hereditary myopathies in adults, and to oculopharyngeal muscular dystrophy (OPMD). Thus, the generalized statement that muscular dystrophies are always associated with high CK levels is not true. In distal myopathies, the degree of hyperCKemia varies widely and is dependent on the cause of the disease. In patients with non-dystrophic myotonias, myofibrillar myopathies and congeni- tal myopathies with specific structural abnormalities, CK levels are typically only slightly to moderately elevated or even within the normal range.

In patients with metabolic myopathies which include glycogenoses, lipid myopathies and mitochondrial myopathies, CK levels again depend very much on subtype and in some cases also on the time of blood collection. For example, type 2 glycogenosis (Pompe disease) is associated with mild, persistent hyperCKemia. In type 5 glycogenosis (McArdle disease), clinically characterized by exercise intolerance with myalgia and contractures, CK levels are in the 4-digit range in many patients. In addition, about half of patients with McArdle disease experience recurrent rhabdomyolysis (see below) associated with acute excessive increase in serum CK activity and myoglobinuria. Attack-like increases in CK levels and myoglobinuria in conjunction with critical pain exacerbations occur with the most common form of lipid myopathies-CPT2 deficiency [10]. Potential triggers include prolonged physical exercise, cold, fever, fasting, a diet low in carbohydrates and rich in fats, treatment with valproate, and the administration of anesthetics. In the symptom-free interval, however, CK levels are typical normal or only slightly elevated.

In idiopathic myositis, mean CK levels are at about $3.000 \mathrm{U} / \mathrm{L}$ $(50 \mu \mathrm{kat} / \mathrm{L})[29,64]$, but can increase to levels above $10.000 \mathrm{U} / \mathrm{L}$ $(166.7 \mu \mathrm{kat} / \mathrm{L})$. By contrast, sporadic inclusion body myositis is associated with usually mild to moderate hyperCKemia. 

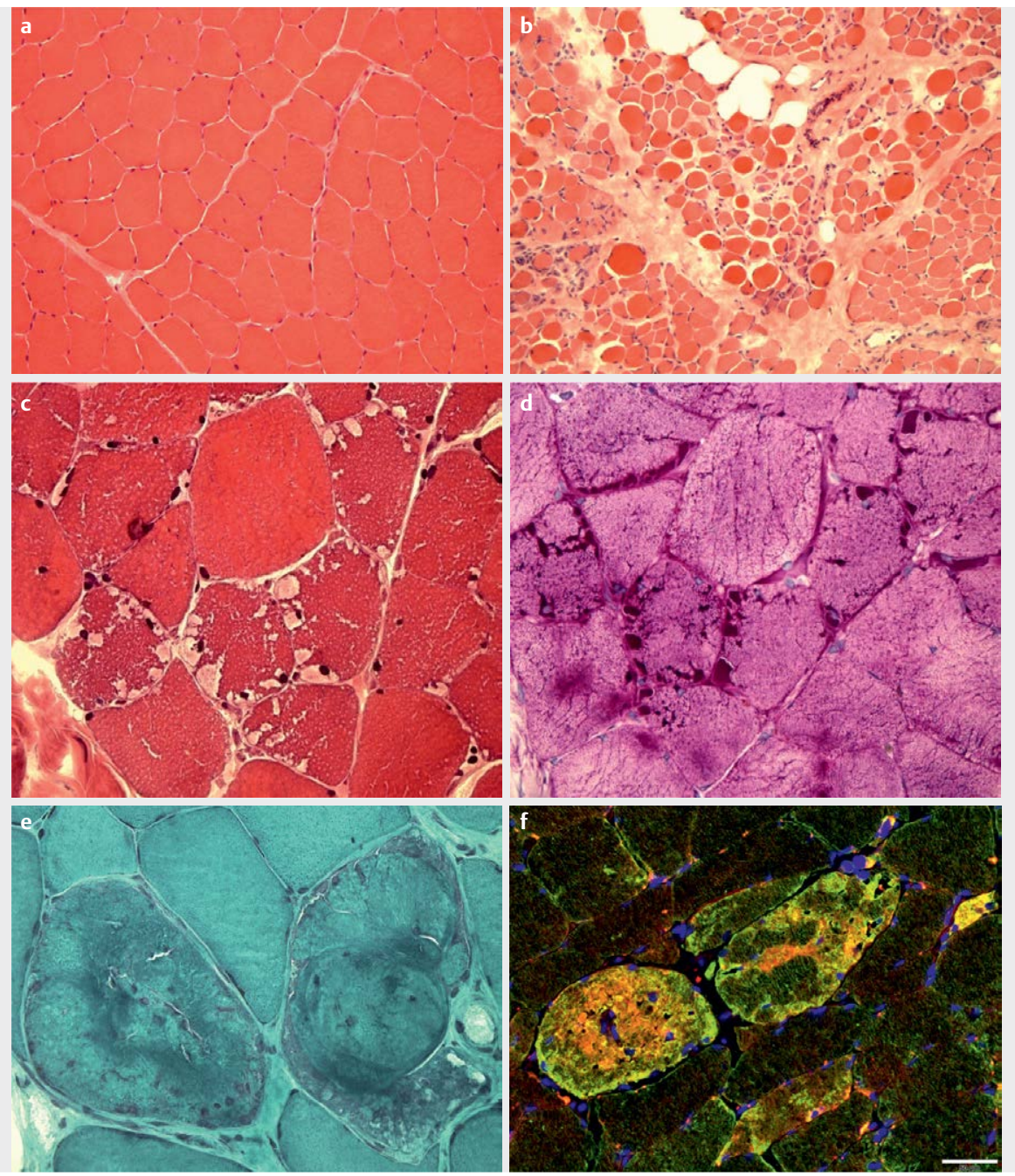

- Fig. 4 Example of histological findings in myopathies. a: Normal control. b: Dystrophic tissue with increased variability of fiber diameters, regenerating muscle fibers, central nuclei, and increased amounts of connective and adipose tissues. $\mathbf{c}$ and $\mathbf{d}$ : glycogenosis type 5 with PAS-positive (d) vacuoles (glycogen deposits). e and $\mathbf{f}$ : Myofibrillar myopathy with massive protein aggregation in muscle fibers and detection of filamin C ( $g r e e n$ in $\mathbf{f}$ ) and desmin (red in f) within the aggregates. a-c: HE stain; d: PAS stain; e: trichrome stain; f: immunofluorescence analyses.

Hypothyroidism-associated myopathy is the most common endocrine myopathy. It may lead to mild hyperCKemia [35] and is clinically characterized by mild, proximal pareses and myalgias as well as slow muscle contraction and delayed muscle relaxation (similar to myotonias, but without myotonic discharges in EMG). These muscle symptoms usually disappear with appropriate therapy. Likewise, hyperthyroidism regularly causes myopathy, but CK levels typically remain within the normal range. However, in rare cases rhabdomyolysis may occur during thyrotoxic crisis.

\section{Statin-associated CK elevation}

Numerous drugs can cause muscle symptoms and hyperCKemia. Due to its clinical relevance, also for neurologists, we will discuss statin-induced CK elevation in detail in the following.

According to a recent review, statin-associated muscle symptoms occur in $7-29 \%$ of patients treated with statins [57]. Myalgia in combination with mild CK elevation is common, but muscle weakness and muscle cramps may occur as well. Symptom onset is typically within the first 4-6 weeks after start of treatment [44] and this side effect is one of the main reasons for discontinuation of cholesterol-lowering statins. In 0.1 to $0.01 \%$ of patients, CK levels increase to more than 10 times the ULN. Risk factors for statin intolerance include age, sex, physical constitution, comorbidities, and co-medication as well as genetic predisposition [58, 2, 49].

The pathophysiology of statin-induced adverse effects is related to mitochondrial dysfunction associated, among others, with changes in coenzym Q10 concentrations in the muscle leading to impaired intracellular ATP generation. Furthermore, alterations in protein homeostasis, including changes in the autophagy system, are discussed [57]. In some patients, autoimmunogenic reactions with generation of antibodies to HMG-CoA reductase have been described [32], potentially leading to independent necrotizing myopathy.

Isolated hyperCKemia does not necessarily require a change of statin medication. In clinically asymptomatic patients, CK levels up to 5 times above the ULN can be tolerated [40-3]. Definitions of 
statin-induced myopathy and recommendations for the management of statin-associated muscle symptoms and hyperCKemia differ $[40,36,57]$ and some have to be questioned from the perspective of myology [3]. Below, a brief summary of the 2015 recommendations of the European Atherosclerosis Society [57]:

In patients with muscle symptoms and CK levels of up to 4 times the (old) ULNs, the first step should be to interrupt statin therapy for 2-4 weeks. If symptoms improve, it is recommended to start treatment with another statin after review of the indication. If symptoms recur, a low-dose therapy with a third statin or adjustment of the administration interval should be attempted. In case symptoms persist despite interruption of statin therapy, another cause of the muscle symptoms can be assumed and it is recommended to restart the same statin therapy. A critical point, howev$\mathrm{er}$, is that it can take several months after statin discontinuation before statin-associated muscle symptoms have disappeared. Taking this into consideration, in our opinion the approach should be to either switch the patient to an alternative LDL cholesterol-lowering treatment or-if reasonable-to interrupt statin treatment for a prolonged period of time and then restart it. In addition, these patients should be referred to a neuromuscular center for further diagnostic work-up.

In case of muscle symptoms and a CK increase by more than 4 times the ULN, first statin treatment should be interrupted for a period of 6 weeks and then an attempt with an alternative low-dose statin medication or an alternating drug administration should be made. In case of findings indicative of statin-induced rhabdomyolysis (see below), however, no further statin treatment should be attempted.

The aim of this management approach is to adequately lower LDL cholesterol levels [47] with statins (up to the maximum dose tolerated by the patient). If this is not possible, treatment with ezetimibe, if necessary in combination with bile acid reabsorption inhibitors and/or fibrates (except for gemfibrozil) is recommended. If the target range of LDL levels is still not reached with this regimen, the use of new treatment options, such as monoclonal anti-PCSK9 antibodies or CETP inhibitors, should be considered.

\section{Neuropathies}

Medical history and clinical examination findings often are strongly indicative of peripheral neuropathy. However, in case of exclusive motor deficits, it may be difficult to distinguish between neuropathy and primary muscle conditions, such as distal myopathies. However, the results of additional electrophysiological testing usually help to overcome this hurdle. In complicated cases, a combination of peripheral neuropathy and myopathy can be present, for example in certain types of myofibrillar myopathies [23] or BICD2-associated disorders [60]. For information about the pragmatic diagnostic work-up of hereditary peripheral neuropathies, please refer to the review by Dräger and Young [12].

In case of acquired and hereditary neuropathies with involvement of the $2^{\text {nd }}$ motor neuron, mild to moderate hyperCKemia is often present. In cohort studies, CK levels were elevated in $27 \%$ of patients with chronic inflammatory demyelinating polyradiculoneuropathy (CIDP, $N=67$ ) [1], in more than $50 \%$ of patients with Guillain-Barré syndrome (GBS, N=21) [51], in $67 \%$ of patients with small-fiber neuropathy $(\mathrm{N}=12)$ [33], and in all patients with axonal neuropathy due to TRPV4 mutations ( $N=17)$ [14]. In Charcot-Marie-Tooth (CMT) disease patients with NEFL mutations, a study found CK levels between 433 and $1213 \mathrm{U} / \mathrm{L}$ (7.2-20.2 $\mu \mathrm{kat} / \mathrm{L})$ [4]. With other types of CMT ( $N=205)$, between $3 \%$ (in PMP22 duplications) and $30 \%$ (with MPZ mutations) of patients had increased CK levels [19]. In a cohort of 504 patients with spinal muscular atrophy (SMA), CK activity with SMA I and II was about one third, in SMA III in $57 \%$ and in SMA IIIb in more than $90 \%$ of patients above twice the standard deviation [52]. In a more recent study with 80 patients with amyotrophic lateral sclerosis (ALS), serum CK activity was increased in $45 \%$ of patients [18]. The highest level in that study was $1992 \mathrm{U} / \mathrm{L}(33.2 \mu \mathrm{kat} / \mathrm{L})$. This is in line with our own clinical experience that CK levels above $1000 \mathrm{U} / \mathrm{L}(16.7 \mu \mathrm{kat} / \mathrm{L})$ are relatively common in patients with ALS. In patients with Kennedy syndrome, CK levels of more than $4000 \mathrm{U} / \mathrm{L}(66,7 \mu \mathrm{kat} / \mathrm{L})$ have been reported $[17,48]$. However, a myopathic component of the disease is being discussed [56]. Typically, CK levels in neuropathy patients are markedly lower and CK levels above $1000 \mathrm{U} / \mathrm{L}(16.7 \mu \mathrm{kat} / \mathrm{L})$ are, in principle, indicative of a myopathy.

\section{Rhabdomyolysis}

Rhabdomyolysis is a potentially life-threatening condition in which content of muscle fibers is released into the circulation after acute and extensive injury to muscle tissue. CK levels increase to more than 10 times the ULN (in some cases > 200,000 U/L (3333.3 $\mu \mathrm{kat} / \mathrm{L}))$, but subsequently drop rapidly. Clinically, patients present with the following key symptoms: severe myalgia and paresis, developed within hours or days and affecting especially the proximal leg muscles; muscle swelling and the reddish-brown urine of myoglobinuria. However, in most cases not all symptoms appear simultaneously. The classical triad of muscle weakness, myalgias and discoloration of urine is rarely seen in patients [65]. The definition of rhabdomyolysis, for which as yet no consensus has been reached, has certainly contributed to this. Some authors call for using a CK elevation of more than 5 times the ULN as the threshold [26]. However, we think this threshold is far too low. It should be noted that the risk of acute renal failure is generally very low if CK levels are under 15 000-20 000 U/L (250-333.3 $\mu \mathrm{kat} / \mathrm{L})$ [5]. Howev$\mathrm{er}$, in the presence of concomitant factors such as sepsis, dehydration and acidosis, renal failure can already occur at CK levels starting from about $5000 \mathrm{U} / \mathrm{L}(83.3 \mu \mathrm{kat} / \mathrm{L})$ [5].

The most important complication of rhabdomyolysis is acute renal failure. It is primarily caused by tubular obstruction by myoglobin casts, but vasoconstriction and hypovolemia as well as direct toxic effects of myoglobin to renal parenchyma can play a role too. Cardiac arrhythmia, disseminated intravascular coagulation (DIC) and compartment syndromes are other relevant complications [65]. 
Recommendations for acute treatment include forced diuresis with intravenous infusions ( $\mathrm{NaCl} 0.9 \%$ ) and furosemide, correction of electrolyte imbalances and, if required, intravenous administration of sodium bicarbonate to alkalize the urine (target pH 6.5). Infusion treatment should be continued until CK levels have dropped to $<1000 \mathrm{U} / \mathrm{L}(<16.7 \mu \mathrm{kat} / \mathrm{L})$. Hemodialysis may be indicated to treat life-threatening hyperkalemia or metabolic acidosis. Compartment syndromes may require surgical treatment and attention should be paid to hemorrhagic complications in patients with DIC [65].

Rhabdomyolysis can be triggered by a wide range of acquired and inherited factors [65]. The most common causes are substance abuse (34\%), medications ( $11 \%$ ), accidents ( $9 \%)$, and seizures (7\%) [37]. Hereditary myopathies should be considered especially in patients with recurrent episodes, positive family history or pre-existing muscular exercise intolerance. They are typically associated with rhabdomyolysis (at times the first manifestation of the disease), comprise some types of metabolic myopathies (e. g., CPT2 deficiency, McArdle disease), certain muscular dystrophies and also structural myopathies.

Functional tests for metabolic myopathies play a key role in the work-up of rhabdomyolysis. These include the non-ischemic forearm exercise test, the bicycle ergometer test and the tandem mass-spectrometric measurement of serum carnitine and acylcarnitine. These tests can provide first non-invasive hints of potential disturbances of glycogen metabolism, lipid metabolism and the mitochondrial respiratory chain. Ideally, these special tests should be conducted in muscle centers experienced in the performance and interpretation of these examinations. It should then be evaluated on an individual basis whether a muscle biopsy and/or additional genetic tests should follow. Currently, the sensitivity of selective NGS panel testing, comprising metabolic and other genes, after a completed rhabdomyolysis episode is scientifically evaluatedand may replace or complement the more invasive and time-consuming muscle biopsy. An important aspect of the diagnostic work-up of rhabdomyolysis is that a muscle biopsy should only be performed at least 4 weeks after a completed episode of rhabdomyolysis so that the histopathological assessment is not complicated by the great number of rhabdomyolysis-related muscle fiber necroses.

\section{Conclusion for Clinical Practice}

Elevated CK levels should first be rechecked after several days of reduced physical activity and alcohol abstinence, using the recommended new reference range. In case of persisting hyperCKemia, the next diagnostic step should be to rule out potential medical causes. Asymptomatic CK elevations do not necessarily require a diagnostic work-up (see algorithm), but clinical follow-ups are advisable and in case of anesthesia substances which may trigger $\mathrm{MH}$ should be avoided, as a precaution. In patients with symptomatic hyperCKemia, it should be taken into consideration that neuropathies with involvement of the $2^{\text {nd }}$ motor neuron are frequently as- sociated with CK elevations, while myopathies are not necessarily associated with high CK levels. In case of suspected neuromuscular disease, further tests should be performed, preferably in a Neuromuscular Center, especially when the phenotype is not specific for a particular disease. Adverse drug reactions are another potential cause of hyperCKemia. In asymptomatic patients with statin-associated CK elevation, CK levels up to 5 times the ULN can be tolerated. In case of statin-associated muscle symptoms, a change in medication is usually indicated; however, in case of rhabdomyolysis, treatment with HMG-CoA-reductase inhibitors should be discontinued permanently. Severe rhabdomyolysis which can be caused by a variety of factors is a potentially life-threatening condition and may lead to acute renal failure. One should think of an underlying myopathy especially in patients with recurrent rhabdomyolyses, a family history positive for muscle disorders or in case of muscular exercise intolerance.

\section{GENETIC TESTING IN ASYMPTOMATIC HYPER-} CKEMIA

Whether it is reasonable to perform molecular genetic analyses in patients with asymptomatic hyperCKemia remains the subject of controversy. For example, the EFNS guidelines recommend to conduct genetic tests in all women with $\mathrm{CK}$ elevations $>325 \mathrm{U} / \mathrm{L}$ ( $>5.4 \mu \mathrm{kat} / \mathrm{L}$ ) to determine carrier status for Duchenne and Becker types of muscular dystrophy caused by mutations in the dystrophin gene (DMD). The sensitivity of the recommended method (multiplex ligation-dependent probe amplification [MLPA]) is reported to be approximately $70 \%$ [25]. However, taking into account the incidence of dystrophinopathies [24], the distribution of CK levels in the healthy population (see above) and the fact that CK levels are not elevated in $33-50 \%$ of carriers [27, 62, 7, 59-13] (based on the current reference range; consequently, the proportion of affected women with CK levels of up to $325 \mathrm{U} / \mathrm{L}(5.4 \mu \mathrm{kat} / \mathrm{L})$ is even higher), the sensitivity of the recommended method to detect carriers of dystrophinopathies is $<50 \%$ with a specificity of $<0.5 \%$. Thus, more than 200 women have to undergo expensive genetic testing to identify one carrier. Besides that, when using this method, more than half of the carriers are not detected. Thus, in this context CK levels are only of limited value as a biomarker. Due to the lack of convincing data, we are critical of this EFNS recommendation; consequently, we have not included it in our algorithm (see $\triangleright$ Fig. 1). In this context, we see preconception carrier screening as a more appropriate approach. Couples with the desire to have a child are tested for carrier status for various severe disorders with autosomal recessive or X-linked inheritance which applies to numerous myopathies. However, ethical aspects need to be taken into consideration [39]. 


\section{MYALGIAS}

In the differential diagnosis of hyperCKemia, asking patients about their history of myalgias is important. Several myopathies have myalgia in combination with hyperCKemia as characteristic-or at least important-features (e.g., in McArdle disease [50] und other metabolic myopathies, certain types of myositis [11], and myotonic dystrophy type 2 [53]). Muscle pain may also play an important role in disorders such as FSHD, dystrophinopathies, LGMD1C, LGMD2A, LGMD2I, and myotonic dystrophy type 1 . Soreness experienced deep in the muscle is characteristic of myopathies. In other types of hyperCKemia, with or without myopathy, patients may experiences myalgia but it plays a minor role (e.g., in Pompe disease [22]), while still other conditions are completely or almost completely painless.

When taking the patient's history, it should be clarified whether the muscle pain is localized or generalized and whether the patient experiences myalgia in its strict sense or cramps, i.e. muscle pain caused by involuntary and usually visible, very painful muscle contractions. In myalgias stricto sensu it should be asked whether the patient experiences the pain at rest or reproducibly linked to physical exercise (exercise intolerance). In patients presenting with the combination of hyperCKemia and myalgias, various causes have to be taken into considerations, above all myopathies, but also neuropathies. An important differential diagnosis of this combination is physical activity or excessive exercising in untrained individuals. As a rule of thumb, muscle pain during or immediately after physical exercise is indicative of myopathy (e. g., metabolic myopathy). By contrast, muscle pain experienced only $24-48$ hours after physical exercise is suggestive of soreness after excessive exercising. The pathophysiology of these myalgias is not fully understood, but apparently microtrauma and local inflammation play an important role. As with hyperCKemia, the patient should first be asked to rest for a few days to be able to better assess the link between muscle pain and physical exercise. By contrast, cramps are of primary neurogenic origin, but may also occur with a number of myopathies and with disorders of the central nervous system and during treatment with certain medications.

\section{Conflict of Interest}

The authors declare no conflict of interest.

\section{References}

[1] Abraham A, Albulaihe $\mathrm{H}$, Alabdali M et al. Frequent laboratory abnormalities in CIDP patients. Muscle Nerve 2016; 53: 862-865

[2] Ahmad Z. Statin intolerance. Am J Cardiol 2014; 113: 1765-1771
[3] Argov Z. Statins and the neuromuscular system: A neurologist's perspective. Eur J Neurology 2015; 22: 31-36

[4] Berciano J, Garcia A, Peeters K et al. NEFL E396K mutation is associated with a novel dominant intermediate Charcot-Marie-Tooth disease phenotype. J Neurol 2015; 262: 1289-1300

[5] Bosch X, Poch E, Grau JM. Rhabdomyolysis and acute kidney injury. N Engl J Med 2009; 361: 62-72

[6] Brewster LM, Mairuhu G, Sturk A et al. Distribution of creatine kinase in the general population: Implications for statin therapy. Am Heart J 2007; 154: 655-661

[7] Cardon MW. 50 years ago in the Journal of Pediatrics: An assessment of the creatine kinase test in the detection of carriers of Duchenne muscular dystrophy. J Pediatrics 2017; 186: 63

[8] Dabby R, Sadeh M, Herman O et al. Asymptomatic or minimally symptomatic hyperCKemia: Histopathologic correlates. Isr Med Assoc J 2006; 8: 110-113

[9] D'Adda E, Sciacco M, Fruguglietti ME et al. Follow-up of a large population of asymptomatic/oligosymptomatic hyperckemic subjects. J Neurol 2006; 253: 1399-1403

[10] Deschauer M, Wieser T, Zierz S. Muscle carnitine palmitoyltransferase II deficiency: Clinical and molecular genetic features and diagnostic aspects. Arch Neurol 2005; 62: 37-41

[11] Dobloug C, Garen T, Bitter H et al Prevalence and clinical characteristics of adult polymyositis and dermatomyositis; Data from a large and unselected Norwegian cohort. Ann Rheum Dis 2015; 74: 1551-1556

[12] Dräger B, Young P. Pragmatische Diagnostik hereditärer Neuropathien. Akt Neurol 2016; 43: 256-266

[13] Dubowitz V. The female carrier of Duchenne muscular dystrophy. $\mathrm{Br}$ Med J (Clin Res Ed) 1982; 284: 1423-1424

[14] Echaniz-Laguna A, Dubourg O, Carlier P et al. Phenotypic spectrum and incidence of TRPV4 mutations in patients with inherited axonal neuropathy. Neurology 2014; 82: 1919-1926

[15] Fernandez C, de Paula AM, Figarella-Branger D et al Diagnostic evaluation of clinically normal subjects with chronic hyperCKemia. Neurology 2006; 66: 1585-1587

[16] Filosto M, Tonin P, Vattemi G et al. The role of muscle biopsy in investigating isolated muscle pain. Neurology 2007; 68: 181-186

[17] Ghosh PS, Lahoria R, Milone M et al. Pearls \& Oysters: HyperCKemia with limb-girdle weakness: Think beyond myopathies. Neurology 2014; 83: e209-e212

[18] Gibson SB, Kasarskis EJ, Hu N et al. Relationship of creatine kinase to body composition, disease state, and longevity in ALS. Amyotroph Lateral Scler Frontotemporal Degener 2015; 16: 473-477

[19] Hattori N, Yamamoto M, Yoshihara T et al. Demyelinating and axonal features of Charcot-Marie-Tooth disease with mutations of myelin-related proteins (PMP22, MPZ and Cx32): A clinicopathological study of 205 Japanese patients. Brain 2003; 126: 134-151

[20] Hørder M, Elser RC, Gerhardt W et al. International federation of clinical chemistry, scientific division committee on enzymes: Approved recommendation on IFCC methods for the measurement of catalytic concentration of enzymes. Part 7. IFCC method for creatine kinase (ATP: creatine N-phosphotransferase, EC 2.7.3.2). Eur J Clin Chem Clin Biochem 1991; 29: 435-456

[21] Joy JL, Oh S]. Asymptomatic hyper-CK-emia: An electrophysiologic and histopathologic study. Muscle Nerve 1989; 12: 206-209

[22] Karabul N, Kruijshaar ME, Schober A et al. Pain in adult patients with Pompe disease. Mol Gen Metab Rep 2014; 1: 139-140

[23] Kley RA, van der Ven Hellenbroich Y, Peter FM et al. Clinical and morphological phenotype of the filamin myopathy: A study of 31 German patients. Brain 2007; 130: 3250-3264 
[24] Koeks Z, Bladen CL, Salgado D et al. Clinical outcomes in Duchenne muscular dystrophy: A study of 5345 patients from the TREAT-NMD DMD Global Database 2017; 4: 293-306

[25] Kyriakides T, Angelini C, Schaefer J et al. EFNS guidelines on the diagnostic approach to pauci- or asymptomatic hyperCKemia. Eur ] Neurol 2010; 17: 767-773

[26] Lane R, Phillips M. Rhabdomyolysis. BM] 2003; 327: 115-116

[27] Lee SH, Lee JH, Lee KA et al. Clinical and genetic characterization of female dystrophinopathy. J Clin Neurol 2015; 11: 248-251

[28] Lev El, Tur-Kaspa I, Ashkenazy I et al. Distribution of serum creatine kinase activity in young healthy persons. Clin Chim Acta 1999; 279: 107-115

[29] Leverenz D, Zaha O, Crofford L] et al. Causes of creatine kinase levels greater than $1000 \mathrm{IU} / \mathrm{L}$ in patients referred to rheumatology. Clin Rheumatol 2016; 35: 1541-1547

[30] Lilleng $\mathrm{H}$, Abeler $\mathrm{K}$, Johnsen $\mathrm{SH}$ et al. Variation of serum creatine kinase (CK) levels and prevalence of persistent hyperCKemia in a Norwegian normal population. The Tromsø Study. Neuromuscul Disord 2011; 21: 494-500

[31] Lilleng $\mathrm{H}$, Johnsen SH, Wilsgaard T et al. Are the currently used reference intervals for creatine kinase $(C K)$ reflecting the general population? The Tromsø Study. Clin Chemistry Laboratory Med 2012; 50: 879-884

[32] Limaye V, Bundell C, Hollingsworth P et al. Clinical and genetic associations of autoantibodies to 3-hydroxy-3-methyl-glutarylcoenzyme a reductase in patients with immune-mediated myositis and necrotizing myopathy. Muscle Nerve 2015; 52: 196-203

[33] Lopate G, Streif E, Harms M et al. Cramps and small-fiber neuropathy. Muscle Nerve 2013; 48: 252-255

[34] Malandrini A, Orrico A, Gaudiano C et al. Muscle biopsy and in vitro contracture test in subjects with idiopathic HyperCKemia. Anesthesiology 2008; 109: 625-628

[35] McGrowder DA, Fraser YP, Gordon L et al. Serum creatine kinase and lactate dehydrogenase activities in patients with thyroid disorders. Niger J Clin Pract 2011; 14: 454-459

[36] McKenney JM, Davidson MH, Jacobson TA et al. Final conclusions and recommendations of the National Lipid Association Statin Safety Assessment Task Force. Am J Cardiol 2006; 97: 89C-94C

[37] Melli G, Chaudhry V, Cornblath DR. Rhabdomyolysis: an evaluation of 475 hospitalized patients. Medicine 2005; 84: 377-385

[38] Moghadam-Kia S, Oddis CV, Aggarwal R. Approach to asymptomatic creatine kinase elevation. Cleve Clin J Med 2016; 83: 37-42

[39] Molster CM, Lister K, Metternick-Jones S et al. Outcomes of an international workshop on preconception expanded carrier screening: some considerations for governments. Frontiers Public Health 2017; 5: 25

[40] Mosshammer D, Schaeffeler E, Schwab M et al. Mechanisms and assessment of statin-related muscular adverse effects. $\mathrm{Br}$ J Clin Pharmacology 2014; 78: 454-466

[41] Nardin RA, Zarrin AR, Horowitz GL et al. Effect of newly proposed CK reference limits on neuromuscular diagnosis. Muscle Nerve 2009; 39: 494-497

[42] Nicholson GA, McLeod JG, Morgan G et al. Variable distributions of serum creatine kinase reference values. Relationship to exercise activity. J Neurol Sci 1985; 71: 233-245

[43] Norwood FLM, Harling C, Chinnery PF et al. Prevalence of genetic muscle disease in Northern England: In-depth analysis of a muscle clinic population. Brain 2009; 132: 3175-3186

[44] Parker BA, Capizzi JA, Grimaldi AS et al. Effect of statins on skeletal muscle function. Circulation 2013; 127: 96-103
[45] Prelle A, Tancredi L, Sciacco M et al. Retrospective study of a large population of patients with asymptomatic or minimally symptomatic raised serum creatine kinase levels. J Neurol 2002; 249: 305-311

[46] Reijneveld JC, Notermans NC, Linssen WH et al. Benign prognosis in idiopathic hyper-CK-emia. Muscle Nerve 2000; 3: 575-579

[47] Reiner Z, Catapano AL, de Backer G et al ESC/EAS Guidelines for the management of dyslipidaemias: The task force for the management of dyslipidaemias of the European Society of Cardiology (ESC) and the European Atherosclerosis Society (EAS). Eur Heart ] 2011; 32: 1769-1818

[48] Rhodes LE, Freeman BK, Auh S et al. Clinical features of spinal and bulbar muscular atrophy. Brain 2009; 132: 3242-3251

[49] Riphagen IJ, van der Veer E, Muskiet FAJ et al. Myopathy during statin therapy in the daily practice of an outpatient cardiology clinic: Prevalence, predictors and relation with vitamin D. Curr Med Res Opin 2012; 28: 1247-1252

[50] Rommel O, Kley RA, Dekomien G et al. Muscle pain in myophosphorylase deficiency (McArdle's disease): The role of gender, genotype, and pain-related coping. Pain 2006; 124: 295-304

[51] Ropper AH, Shahani BT. Pain in Guillain-Barre syndrome. Arch Neurol 1984; 41: 511-514

[52] Rudnik-Schoneborn S, Lutzenrath S, Borkowska J et al. Analysis of creatine kinase activity in 504 patients with proximal spinal muscular atrophy types I-III from the point of view of progression and severity. Eur Neurol 1998; 39: 154-162

[53] Schneider C, Reiners K, Toyka KV. Myotone Dystrophie (DM/ Curschmann-Steinert-Erkrankung) und proximale myotone Myopathie (PROMM/Ricker-Syndrom). Myotone Muskelerkrankungen mit multisystemischen Manifestationen (Myotonic dystrophy (DM/ Curschmann-Steinert disease) and proximal myotonic myopathy (PROMM/Ricker syndrome). Myotonic muscle diseases with multisystemic manifestations). Nervenarzt 2001; 72: 618-624

[54] Schumann G, Bonora R, Ceriotti F et al. IFCC primary reference procedures for the measurement of catalytic activity concentrations of enzymes at 37 degrees $C$. Part 2. Reference procedure for the measurement of catalytic concentration of creatine kinase. Clin Chem Lab Med 2002; 40: 635-642

[55] Simmons Z, Peterlin BL, Boyer P] et al. Muscle biopsy in the evaluation of patients with modestly elevated creatine kinase levels. Muscle Nerve 2003; 27: 242-244

[56] Soraru G, D’Ascenzo C, Polo A et al. Spinal and bulbar muscular atrophy: Skeletal muscle pathology in male patients and heterozygous females. J Neurol Sci 2008; 264: 100-105

[57] Stroes ES, Thompson PD, Corsini A et al. Statin-associated muscle symptoms: Impact on statin therapy-European Atherosclerosis Society Consensus Panel Statement on Assessment, Aetiology and Management. Eur Heart J 2015; 36: 1012-1022

[58] Talameh JA, Kitzmiller JP. Pharmacogenetics of statin-induced myopathy: a focused review of the clinical translation of pharmacokinetic genetic variants. J Pharmacogenomics Pharmacoproteomics 2014, doi:10.4172/2153-0645.1000128

[59] Thompson MW, Murphy EG, McAlpine PJ. An assessment of the creatine kinase test in the detection of carriers of Duchenne muscular dystrophy. J Pediatrics 1967; 71: 82-93

[60] Unger A, Dekomien G, Guttsches A et al. Expanding the phenotype of BICD2 mutations toward skeletal muscle involvement. Neurology 2016; 87: 2235-2243

[61] Urdal P, Urdal K, Strømme JH. Cytoplasmic creatine kinase isoenzymes quantitated in tissue specimens obtained at surgery. Clin Chemistry 1983; 29: 310-313 
[62] Villamar López M, Azpeitia González J, Ayuso García C et al. Modificación del cálculo de riesgo en posibles mujeres portadoras de distrofia muscular de Duchenne (DMD) basado en niveles de CPK (Modification of the calculation of risk factors in women, possible carriers of Duchenne muscular dystrophy, based on CPK levels). An Esp Pediatr 1992; 37: 191-194

[63] Weglinski MR, Wedel DJ, Engel AG. Malignant hyperthermia testing in patients with persistently increased serum creatine kinase levels. Anesth Analg 1997; 84: 1038-1041
[64] Zhang Y, Huang J, Wang Z et al. Value of muscle enzyme measurement in evaluating different neuromuscular diseases. Clin Chim Acta 2012; 413: $520-524$

[65] Zutt R, van der Kooi AJ, Linthorst GE et al. Rhabdomyolysis: review of the literature. Neuromuscul Disord 2014; 24: 651-659 